\title{
In-situ TEM Investigation on Reaction Mechanisms of Conversion Electrode Materials for Batteries
}

Dong $\mathrm{Su}^{1, *}$

${ }^{1}$ Center for Functional Nanomaterials, Brookhaven National Laboratory, Upton, United States

* Corresponding author: dsu@bnl.gov or free.sd@gmail.com

Lithium ion batteries (LIBs) have dominated portable electronics and are penetrating the markets of electric vehicles. Current electrode materials have specific capacities ranged only from 140 to $200 \mathrm{mAh}$

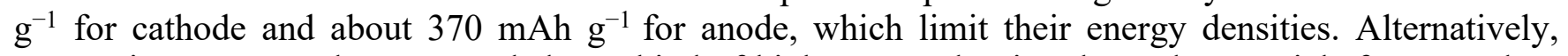
conversion compounds are regarded as a kind of high-energy density electrode materials for secondary ion batteries. However, these compounds suffer a severe voltage hysteresis and a poor cycling stability. These problems have been believed to be intrinsic nature of the conversion reaction chemistry, and the hope of using conversion reaction materials in the next-generation lithium batteries waned. As electrochemical properties are highly dependent on how these complicated reactions proceed, in situ investigation of the reaction pathways is of importance to understand their intrinsic reaction nature.

In this talk, I would review the research frontier of the in situ transmission electron microscopy (TEM) study on the dynamic reaction behavior of conversion metallic compounds, e.g. oxides, fluorides and sulfides, especially on the works using the dry-cell approach in my group [1-8]. Real-time structural evolutions induced by lithium ion's insertion are reflected from in-situ electron diffraction, and thus, from the appearance and disappearance of peaks, we could distinguish reaction steps and possible intermediate phases. On the other hand, in situ TEM or scanning-TEM imaging can help us to understand the phase evolution in real space. In past years, we developed a strain sensitive-STEM imaging approach to visualize the phase boundary movement induced by $\mathrm{Li}$ ions' insertion in $\mathrm{Fe}_{3} \mathrm{O}_{4}, \mathrm{Co}_{3} \mathrm{O}_{4}$ and $\mathrm{SnS}_{2}$. Using in-situ TEM techniques, we investigated the phase evolutions and intermediate phases at non-equilibrium states in conversion compounds during discharging. With the help of X-ray characterizations and DFT calculations, we corroborated information from real-time TEM observation with the performances. Our results may provide new insights into designing conversion-type electrode materials for applications [9].

References:

[1] Kai He, et al., Nano Lett. 15, (2015) p.1437

[2] Kai He, et al, Nano Lett. 15, (2015) p.5755

[3] Kai He, et al., Nature Communications, 7, (2016) p.11441

[4] Xiulin Fan, et al., Nature Communications 9, (2018) p.2324

[5] Sooyeon Hwang, et al. Angew. Chem. Int. Ed 56, (2017) p.7813

[6] Sooyeon Hwang, et al., ACS Nano, 12, (2018) p.3638

[7] Jing Li, et al., ACS Nano, 10, (2016) p.9577

[8] Jing Li, et al., Nature Communications, 10, (2019) 93

[9] This work is supported by the Center for Functional Nanomaterials, Brookhaven National Laboratory, which is supported by the U.S. Department of Energy (DOE), Office of Basic Energy Science, under Contract No. DE- SC0012704. I would like to acknowledge Drs. Kai He, Sooyeon Hwang, Jing Li, Kim Kisslinger, Yimei Zhu and Eric Stach for their help on experiments. 

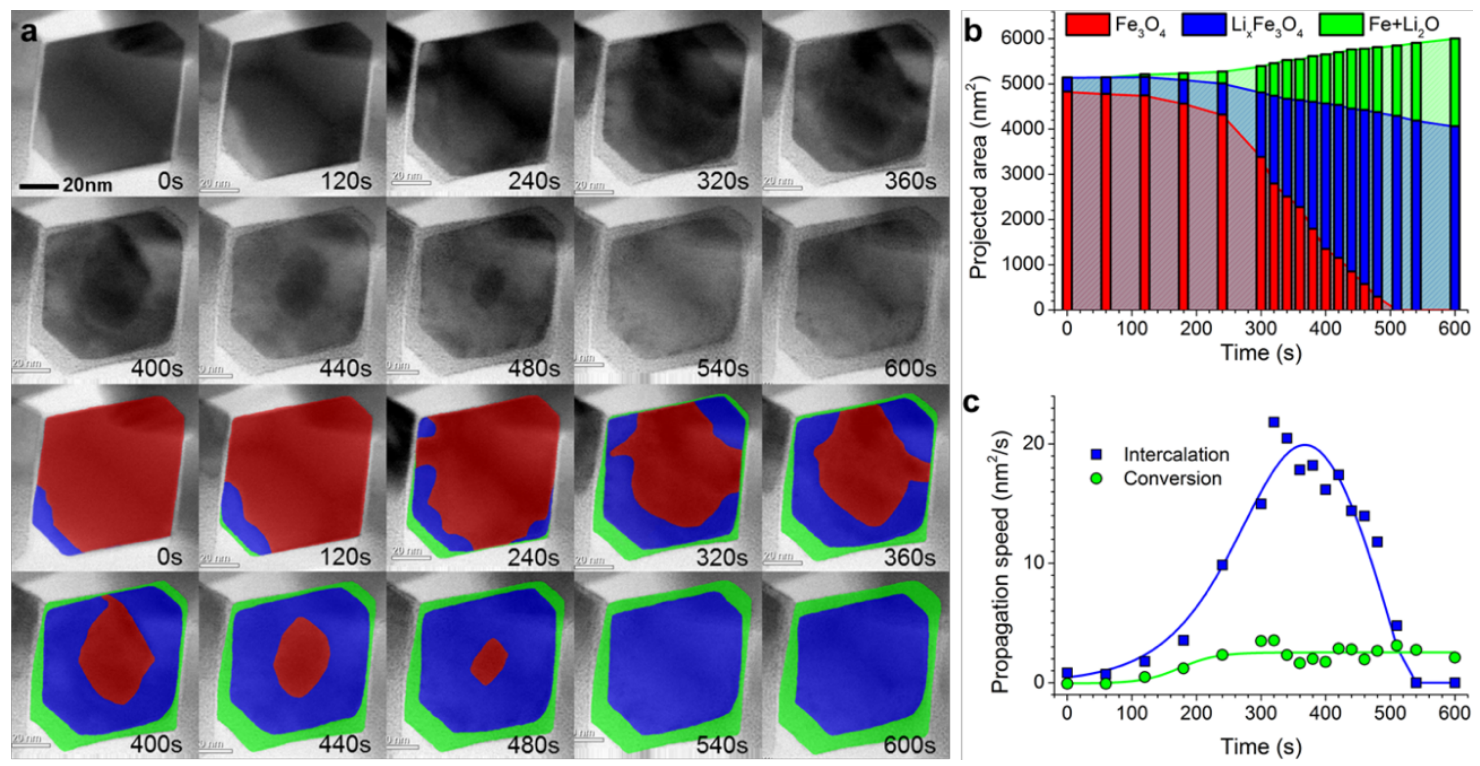

Figure 1. In situ observation of two-step phase transformation during lithiation process [3]. (a) BF-STEM image series showing phase evolution upon lithiation. The overlaid false colors indicate different phases: pristine $\mathrm{Fe}_{3} \mathrm{O}_{4}$ (red), $\mathrm{Li}$-inserted $\mathrm{LixFe}_{3} \mathrm{O}_{4}$ (blue), and $\mathrm{Fe}+\mathrm{Li}_{2} \mathrm{O}$ composition after conversion (green). (b) Projected areas of the three phases as a function of time. (c) Propagation speed of intercalation and conversion as a function of time.
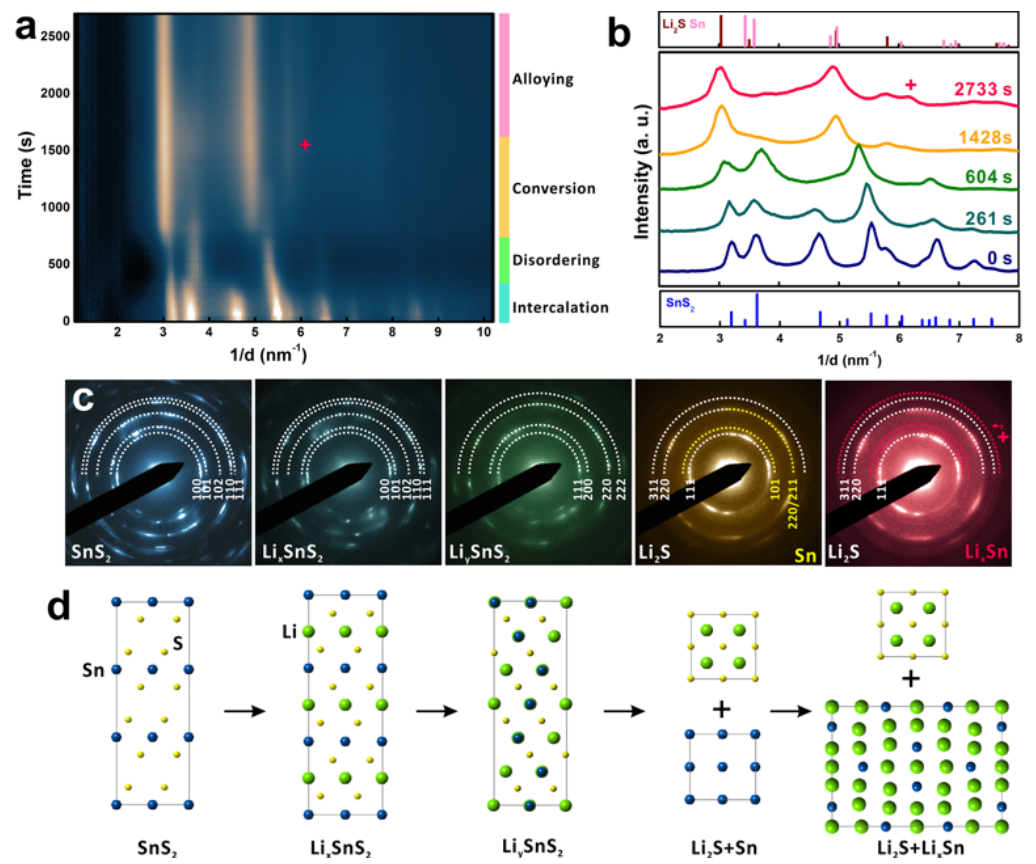

Figure 2. In situ selected area electron diffraction patterns of $\mathrm{SnS}_{2}$ during lithiation [6]. (a) Electron diffraction intensity profile as a function of reaction time during in situ lithiation of $\mathrm{SnS}_{2}$. + denotes the evolution of Li-Sn alloy phase. (b) Radial intensity profiles of diffraction patterns at certain time. (c) Diffraction patterns corresponds to intensity profiles at (b). (d) Atomic models representing phase evolution during lithiation. 